\title{
Coberturas financieras con derivados y su incidencia en el valor de mercado en empresas colombianas que cotizan en Bolsa
}

\author{
Financial hedging with derivatives and its impact on the Colombian \\ market value for listed companies \\ César Augusto Giraldo-Prieto ${ }^{\mathrm{a}, *}$, Gabriel Jaime González Uribe ${ }^{\mathrm{b}}$, \\ Cristhian Vesga Bermejo ${ }^{c}$ y Diana Carolina Ferreira Herrera ${ }^{\mathrm{d}}$ \\ ${ }^{a}$ Universidad de Antioquia, Colombia \\ ${ }^{\mathrm{b}}$ Instituto Tecnológico Metropolitano, Colombia \\ ${ }^{c}$ Universidad Autónoma de Bucaramanga, Colombia \\ ${ }^{\mathrm{d}}$ Universidad Piloto de Colombia, Colombia
}

Recibido el 22 de septiembre de 2016; aceptado el 28 de abril de 2017

Disponible en Internet el 1 de noviembre de 2017

\section{Resumen}

La teoría financiera (Modigliani \& Miller, 1958) plantea la gestión del riesgo como un asunto sin importancia debido a que los mismos accionistas hacían su gestión de cobertura diversificando sus portafolios; sin embargo, estudios posteriores entran en conflicto con dicha premisa y evidencian que la cobertura financiera empresarial mejora el desempeño e incrementa el valor de las mismas (Ahmed, Azevedo y Guney, 2014; Allayannis y Ofek, 1998; Allayannis y Weston, 2001). La gestión eficiente de riesgos de mercado se apoya en los derivados financieros y exige administradores estratégicos y eficientes en cobertura que agreguen valor, especialmente frente a choques y desequilibrios macroeconómicos y financieros. Evidencia empírica analiza el comportamiento de la Q-Tobin como indicador del efecto de las estrategias de cobertura de tipo de cambio asociado al valor de mercado. Este trabajo pretende encontrar evidencia en Colombia sobre el efecto del uso de derivados en el valor de mercado de la empresa. Su valor agregado radica en el análisis que se hace por sectores económicos, identificados por códigos CIIU y agrupados en 5 sectores macro (agropecuario, comercial, industrial o manufactura, servicios y construcción). La metodología empleada incluye la

\footnotetext{
* Autor para correspondencia.

Correo electrónico: cesar.giraldop@udea.edu.co (C.A. Giraldo-Prieto).

La revisión por pares es responsabilidad de la Universidad Nacional Autónoma de México.
} 
estimación de varios modelos de regresión en panel de datos, utilizando una regresión Pooled con estimadores de efectos fijos y aleatorios mediante el estimador de máxima verosimilitud. En general, se encontró una prima por cobertura estadística y financieramente significativa para empresas expuestas a riesgos de tipo de cambio que usan derivados de un $6.3 \%$ de promedio sobre el valor de mercado. Adicionalmente, se encontraron resultados mixtos con relación a las variables analizadas en el modelo.

(C) 2017 Universidad Nacional Autónoma de México, Facultad de Contaduría y Administración. Este es un artículo Open Access bajo la licencia CC BY-NC-ND (http://creativecommons.org/licenses/by-nc-nd/4.0/).

Palabras clave: Coberturas financieras; Instrumentos derivados; Riesgo de mercado; Valor de mercadoG320; G230; G130

\section{Abstract}

The financial theory (Modigliani \& Miller, 1958) rises that risk management was not an issue for companies because shareholders could make their own hedging management through portfolio diversification; however, further studies conflict with that statement and show that corporate financial hedging improves performance and increases the value thereof (Ahmed, Azevedo, \& Guney, 2014; Allayannis \& Weston, 2001; Allayannis $\&$ Ofek, 1998). Efficient management of market risks, which is based on the use of financial derivatives, demands strategic and efficient managers in hedging that adds value to the firm, especially in against shocks and imbalances from a macroeconomic and financial nature. Empirical evidence analyzes the performance of the Q-Tobin as an indicator of the effect of hedging strategies of exchange rate associated to the market value. This paper aims to find evidence in Colombia on the effect of using derivatives in the market value of the firm. Its added value lies in the analysis made by economic sectors, identified by CIIU codes and grouped into 5 sectors (Agricultural, Commercial, Industrial or Manufacturing, Services and Construction). The methodology includes several models estimating regression panel data, using a Pooled regression with estimators of fixed and random effects by maximum likelihood estimator. In general, it was found a premium due to hedging, statistically and financially significant, for companies exposed to exchange rate risks that use derivatives by an average of $6.3 \%$ on the market value. Moreover, mixed results were found regarding the analyzed variables in the model.

(C) 2017 Universidad Nacional Autónoma de México, Facultad de Contaduría y Administración. This is an open access article under the CC BY-NC-ND license (http://creativecommons.org/licenses/by-nc-nd/4.0/).

Keywords: Financial hedging; Financial derivatives; Market risk; Market value

JEL classification: G320; G230; G130

\section{Introducción}

Las dinámicas de la economía contemporánea, del comercio moderno y el desarrollo de los mercados financieros, traen consigo altas volatilidades que exigen mayor velocidad de adaptación y capacidad administrativa por parte de los gerentes modernos, quienes en procura de maximizar el valor de la empresa y evitar la quiebra de la misma, necesariamente deberán plantearse estrategias que les permitan apropiarse de herramientas de gestión y mitigación del riesgo, en las que los derivados financieros juegan un papel crucial, en la medida en que las empresas se apropian de estos instrumentos con conocimiento de los diferentes productos y estrategias de cobertura, que se aplican en la medida en que el mercado de derivados logre un mayor desarrollo (Sierra González y Londoño Bedoya, 2010). 
Colombia inició operaciones con derivados financieros en mercados de Bolsa hace menos de una década $\mathrm{y}$, aunque presenta rezagos frente a otros países ${ }^{1}$, es notable su aceptación en los últimos años, reflejada en un crecimiento de un $57 \%$ de los volúmenes contratados de junio de 2015 a junio de 2016. Este puede estar asociado a la inversión extranjera directa (IED) tanto hacia dentro como hacia afuera (Alvarado Cardozo, Rassa Robayo y Rojas Moreno, 2014), y al aumento de las necesidades de cobertura de empresas del sector real que se involucran cada vez más en las actividades y operaciones de comercio internacional.

Desde el momento en que se da origen a los derivados financieros y se abren las puertas en los mercados de capitales para su uso, nace la inquietud sobre la efectividad real de estos en las finanzas corporativas, tanto en la mitigación de riesgo como en el aumento en el valor de las empresas y la sostenibilidad de las mismas, especialmente en economías emergentes, que son más susceptibles a choques externos y desequilibrios domésticos en términos de intercambios comerciales asociados a volatilidades de las tasas de cambio. Con respecto a los países emergentes, Caballero (2003) plantea que es necesario el acceso a coberturas e instrumentos de aseguramiento que protejan contra eventos desastrosos causados por las volatilidades en los flujos de capitales. Por ende, las coberturas son vitales para la sostenibilidad económica y la viabilidad financiera de una organización.

Uno de los aspectos relevantes en el estudio de derivados es la relación con el nivel de endeudamiento de las empresas, de forma que la influencia ejercida por la cobertura sobre el costo de la deuda, se da principalmente a través de la baja probabilidad de riesgo de quiebra y costos de agencia, y reducción de las asimetrías de información (Chen y King, 2014); no obstante, si la empresa cuenta con alto endeudamiento, el efecto podría ser contrario. Se espera entonces que si el país en su conjunto (inversionistas, empresas y Gobierno) participan activamente del Mercado de Capitales con coberturas para la mitigación de riesgo de tipo de cambio, se mejore la estabilidad económica, principalmente si la exposición está ligada al endeudamiento en moneda extranjera. Para Allayannis, Lel y Miller (2012), quienes investigaron la relación entre el valor de mercado de las empresas y la cobertura con derivados, los resultados son variados y dependen del contexto económico y sus características, como por ejemplo: el tipo de empresa, las características del mercado financiero y el nivel de desarrollo del mercado de instrumentos derivados (liquidez, profundidad, diversificación); es así como la investigación en este campo se ha centrado en la Q-Tobin como indicador del valor de mercado de las empresas, apoyada en diferentes modelos multivariados, buscando encontrar evidencia significativa en dicha relación en empresas que cotizan en el Mercado Público de Valores.

Este trabajo de investigación está inmerso en un proyecto más amplio construido por etapas, donde el objetivo inicial es identificar los sectores y empresas que mitigan su riesgo de tipo de cambio por medio de instrumentos derivados en Colombia, y la relación de esta cobertura con el valor de la empresa. En línea con investigaciones empíricas previas (Ahmed, Azevedo y Guney, 2014; Allayannis et al., 2012; González, León y Leiton Rodríguez, 2009; Kapitsinas, 2008; Nelson, Moffitt y Affleck Graves, 2005; Vivel Búa, Otero González, Fernández López y Durán Santomil, 2015), se define la Q de Tobin como variable de interés, indagando sobre la relación del efecto de las coberturas en las empresas y la contribución de estos con la disminución de la exposición cambiaria y con el aumento en el valor de la empresa.

\footnotetext{
1 Informe Mensual Mercados de la Bolsa de Valores de Colombia (BVC), junio 2016. Bolsa de Valores de Colombia. Disponible en: https://www.bvc.com.co/pps/tibco/portalbvc/Home/Mercados/informesbursatiles?action=dummy
} 


\section{Marco teórico}

La administración financiera moderna ha venido reconociendo la importancia de la gestión de los riesgos financieros dentro de sus estrategias corporativas, y los ha incluido dentro de sus tácticas de cobertura, entendiendo que a ella se asocian los buenos resultados empresariales. En México, Munoz Martinez (2016) analiza la importancia de hacer de las coberturas una política de gobierno con el fin de preservar los presupuestos públicos y aumentar las reservas en moneda extranjera. Géczy, Minton y Schrand (1997) realizaron un estudio con 372 empresas industriales de la lista Fortune 500 para examinar actividades de coberturas de divisas con derivados y a la vez contrastar las teorías de cobertura existentes; encontraron que las empresas que realizan estas actividades tienen una relación positiva con las oportunidades de crecimiento. Trabajando con modelos multivariados, se probó que las empresas con crecimiento alto y financiamiento limitado son más propensas a realizar coberturas con derivados de divisas, mejorando sus costos relacionados con restricciones financieras, lo que permite presentar un flujo de caja más ajustado a las oportunidades de inversión y crecimiento, beneficiando principalmente a empresas con mayores volatilidades en sus resultados asociados a mayores riesgos de tipo de cambio asumidos; encontraron además que las empresas usan los derivados de divisas para reducir problemas de subinversión.

Azofra-Palenzuela y Díez Esteban (2001) indican que la implementación de estrategias de cobertura de riesgos promueven la reducción y control de los mismos, y de esta manera permiten evitar futuras quiebras, ya que al estabilizar la volatilidad de los flujos, se disminuye la probabilidad de enfrentar problemas financieros relacionados con el pago de intereses y de deudas.

Algunos elementos importantes que una empresa ha de considerar al momento de aplicar coberturas con derivados son: i) costos de financiamiento, ii) impuestos corporativos, iii) costos de agencia, iv) imperfecciones del mercado, e incluso v) convicción de la gerencia como estrategia de administración del riesgo. Sobre la gestión del riesgo, Hammoudeh y McAleer (2013) mencionan que es un asunto fundamental para la administración óptima de portafolios, y destacan la expansión y crecimiento que han tenido estos instrumentos en los últimos años como área importante de las finanzas. Jin y Jorion (2006) teorizan dos aspectos intentando investigar y explicar por qué los administradores gestionan el riesgo; i) consideran que en línea con la búsqueda de la maximización del valor del accionista, se pretende mitigar la variación de los flujos de caja, y ii) plantean que las coberturas pueden servir como señales de la capacidad de gestión de los administradores, la cual está en sintonía con la maximización de los beneficios de los directivos.

Otros autores asocian las imperfecciones del mercado de capitales con las diferencias en los costos de adquisición de recursos externos o con respecto a costos de transacción y las economías de escala (Froot, Scharfstein y Stein, 1993; Géczy et al., 1997; Smith y Stulz, 1985), por consiguiente, la estrategia de cobertura corporativa genera ventajas asociadas a las estrategias de endeudamiento y crecimiento. Pero la motivación para realizar coberturas por parte de las empresas también depende de elementos como la exposición al tipo de cambio que tiene la compañía y los costes en la gestión del riesgo. Géczy et al. (1997) a su vez propone tres factores que son claves a la hora de decidir una estrategia con operaciones de cobertura con derivados: incentivos existentes para usarlos, el nivel de exposición al tipo de cambio (especialmente) y los costos de implementación de las estrategias de coberturas. Para Froot et al. (1993), las imperfecciones de los mercados de capital y la información asimétrica encarecen el financiamiento.

Otros asumen que la gerencia implementa coberturas debido a la existencia de asimetrías de información que deben ser controladas mediante la transmisión de resultados y habilidades 
gerenciales que aporten herramientas de valoración al mercado de capitales (Bessembinder, 1991; Smith y Stulz, 1985), e identifican la cobertura corporativa como un instrumento en la reducción de costos de transacción. Al respecto, Bartual Sanfeliu y Moya Clemente (2008) relacionan la estrategia de implementación de cobertura con derivados con oportunidades para reducir dificultades financieras, y resaltan que, en empresas con alta deuda y oportunidades de crecimiento, existen mayores probabilidades de establecer programas de cobertura; Froot et al. (1993) destacan que cubrirse contribuye con la disminución de las variaciones de capital necesarias para gastos, inversiones y financiamiento externo costoso, y enuncian cuatro razones para hacer gestión de riesgos en empresas vía coberturas financieras: gerenciales, impuestos, capacidad de endeudamiento e imperfección del mercado de capitales.

Dufey y Srinivasulu (1983) resaltan que la aversión al riesgo de gerentes con participación económica en las empresas que optan por hacer coberturas con derivados para mitigar los riesgos como la volatilidad de los flujos de caja, y por ende su riqueza personal, encuentran un beneficio en ese tipo de estrategias. En los administradores, la aversión al riesgo se ve reflejada en la utilidad y por consiguiente en la volatilidad del beneficio esperado (Smith y Stulz, 1985), con una relación positiva existente entre el uso de los derivados y la riqueza empresarial. DeMarzo y Duffie (1995), en Bartram, Brown y Conrad (2011), intentan explicar la relación proporcional directa entre incentivos y reputación empresarial, e identifican que el accionista se beneficia de las coberturas de riesgo dado que los administradores y/o gerentes cuentan con información no pública y no observable que incide en los flujos de la empresas; contrario a lo propuesto por Modigliani y Miller (1958).

Algunos estudios (Bartram, 2008; Vivel Búa, Otero González, Fernández López y Durán Santomil, 2013) se centran en la industria manufacturera e intentan estudiar el papel de los derivados como instrumento de gestión y cobertura de riesgos; además, las principales razones por la que se usan, el grado de conocimiento de los derivados más usados y las características típicas de las empresas que los utilizan; algunos estudios recientes cuestionan el valor de la cobertura y para ello estudian la gestión de las opciones sobre acciones y la prima de cobertura encontrando que cuando la cobertura está basada en incentivos vía opciones para los gerentes el valor de la empresa decrece (Hagelin, Holmen, Knopf y Pramborg, 2007).

Sierra González y Londoño Bedoya (2010) estudian las coberturas por medio de instrumentos derivados en empresas del sector real (industria y comercio) enfocado en el uso de estos desde que se abrió este mercado en la Bolsa de Valores de Colombia. Para ello, aplican un modelo de regresión logística y establecen dos hipótesis, en línea con estudios previos internacionales, en las que relacionan el uso de los derivados en empresas con sofisticación financiera y estrés financiero, así como la combinación de ellas. Magner Pulgar y Lavín Salazar (2012) estudiaron 201 firmas de la industria de agronegocios en Estados Unidos y encontraron que el $41 \%$ de la muestra hace cobertura de riesgos con derivados, y que empresas con mayores oportunidades de inversión, apalancamiento y músculo financiero presentaron mayores participaciones en este. Los autores emplearon modelos univariados y multivariados con pruebas de robustez, intentando comprender la mecánica de la gestión del riesgo.

Bartram et al. (2011) estudiaron el uso de derivados como medida de riesgo y valor en 47 países, con una muestra de 6888 empresas no financieras; aplicaron test univariados y evidenciaron que el uso de derivados como cobertura es más recurrente en empresas con mayor exposición en commodities, moneda extranjera y tasas de interés, teniendo en cuenta que estas empresas tienen un nivel inferior de riesgo sistémico. A su vez realizaron una comparación entre compañías a partir de operaciones con derivados y aquellas que no, teniendo en cuenta que existe una fuerte diferencia ya que estas últimas muestran una alta volatilidad en los flujos de caja y riesgo sistémico, lo cual 
sugiere que las empresas no financieras en general emplean el uso de derivados con un motivo principal: reducción del riesgo.

Los instrumentos derivados mejoran el desarrollo de los mercados financieros, contribuyen con el aumento de la profundidad y la liquidez al ampliar la base de participantes y brindar diferentes instrumentos y opciones de inversión y cobertura. En los países emergentes, aunque este mercado es incipiente, comienza a crecer notablemente en volúmenes transados al ampliar el mercado OTC al de Bolsa, con lo cual, las empresas de diversos sectores económicos, y los inversionistas en general, encuentran nuevas alternativas de cobertura, inversión, especulación y arbitraje. Lo anterior requiere la sinergia de diferentes instituciones en pro del desarrollo y la diversificación del mercado de instrumentos derivados.

\section{Metodología y descripción de la muestra}

El presente estudio está influenciado por Allayannis et al. (2001) en su aspecto metodológico. Busca analizar si las empresas expuestas a riesgo de moneda extranjera que usan los derivados como estrategia de cobertura, obtienen un beneficio vía Q de Tobin. La investigación es de corte empírico, apoyada en modelos de regresión.

Para ello se seleccionaron 39 empresas, que fueron objeto de estudio estadístico. El proceso inició con 195 empresas inscritas en la Bolsa de Valores de Colombia, luego se identificaron aquellas empresas que hacen parte del sector real y están involucradas en operaciones con derivados financieros. Se tuvo en cuenta información de estados financieros disponibles en el Sistema Integral de Información del Mercado de Valores (en adelante, SIMEV) ${ }^{2}$ de la Superintendencia Financiera de Colombia. Se descartaron las empresas del sector financiero, las aseguradoras y los patrimonios autónomos, debido a que algunas hacen parte del mercado intermediado de instrumentos derivados en Colombia, otros son creadores de mercado, y por ende, en ambos casos, hacen uso de este tipo de instrumentos con propósitos diferentes a los de las empresas del sector real. La muestra se conformó por empresas con información financiera disponible en el SIMEV desde el último trimestre de 2008 hasta el mismo trimestre de 2014: se decidió trabajar con información trimestral para darle volumen a la información y conformar la base de datos con la que se trabajan los modelos; se recolectó información financiera que está dada en millones de pesos colombianos; se decidió iniciar desde el 2008 considerando que, en Colombia, el mercado centralizado de derivados inició transacciones a partir del tercer trimestre de ese año; adicionalmente, en algunos casos se extractaron datos desde los mismos estados financieros reportados en las páginas web de las empresas, y se acudió a las notas de los mismos; adicionalmente se realizaron controles de validación de la información, cruzándola con la base de datos de Economática para brindarle confiabilidad al panel de datos.

Se tenía previsto trabajar con 40 empresas pero una de ellas presentaba valores atípicos extremos y extraños en su Q de Tobin. Se volvió a buscar la información pero fue difícil confirmar los valores, por ser una empresa de nula bursatilidad. Se decidió eliminarla del todo de la muestra para quedar finalmente con 39 unidades de corte transversal. Se obtuvieron un total de 975 observaciones. A diferencia de Allayannis et al. (2001), no se excluyeron las empresas públicas como Ecopetrol, porque aunque puedan ser - o no- duramente reguladas, como lo mencionan los autores, son empresas que optan por coberturas con derivados como parte de su estrategia de

\footnotetext{
2 https://www.superfinanciera.gov.co/jsp/loader.jsf?1Servicio=Publicaciones\&lTipo=publicaciones\&lFuncion=load ContenidoPublicacion $\&$ id $=80102$
} 
negocio. En este sentido, la investigación no pretende identificar el tipo de instrumento derivado utilizado como cobertura, sino identificar si las empresas que se cubren con derivados financieros incrementan su valor respecto de las que no, así como el comportamiento de otras variables. Se consideraron «empresas que usaron derivados» todas aquellas que de una u otra manera se vieron involucradas en operaciones de comercio exterior, o que manifestaron en informes financieros y notas a los estados que tenían posiciones en este tipo de instrumento. En muchos casos se encontró que la empresa informó sobre el uso de derivados como cobertura, mas no informó cifras; es por esto que se trabaja con una variable que permita medir inicialmente la exposición al riesgo de tipo de cambio que tiene la empresa en sus operaciones normales de negocio, relacionando las ventas en moneda extranjera con los ingresos operaciones totales.

\section{Caracterización de las variables dependientes}

La estructura de la base de datos es de tipo panel y vincula como variables independientes las siguientes: tamaño de la empresa, apalancamiento, ingresos operacionales, ventas totales, ventas en el extranjero, crecimiento de la inversión, diversificación geográfica, uso de derivados y pago de dividendos. Estas variables fueron seleccionadas de manera consecuente con estudios que persiguen el mismo objetivo (Allayannis \& Weston, 2001; Bartram, Brown, \& Fehle, 2009; Jin \& Jorion, 2006); considerando además que la mayoría de las empresas usan derivados con fines de cobertura y no con propósitos especulativos, se ha encontrado que el uso de los derivados de tasa de cambio reducen significativamente la exposición que enfrentan las empresas a este tipo de riesgo y que la decisión de usar los derivados depende de los factores de exposición como por ejemplo las ventas extranjeras y el comercio internacional y, otras variables ampliamente asociadas a teorías de cobertura optima como el tamaño y la inversión o los gastos de Investigación y Desarrollo (Allayannis \& Ofek, 1998) En la tabla 1 se muestra un resumen de las variables involucradas en el modelo.

\section{Caracterización de la $Q$ de Tobin como variable independiente}

Para saber si la cobertura aumenta el valor de la empresa, es indispensable analizar cada una de las variables que impacta dicho valor que está representado por la $\mathrm{Q}$ de Tobin $(\mathrm{Q})^{3}$. Esta última es un ratio financiero que mide la relación entre el valor de mercado de la empresa y su valor contable. De esta forma, un valor de esta razón superior a la unidad indica un aumento del valor de la empresa y sugiere un beneficio marginal positivo de nuevas inversiones. Según Fierros Villanueva (2012), este ratio financiero refleja el valor que le da el mercado a la empresa a partir del costo de reposición. Para Montoro y Navarro (2010) en Fierros Villanueva (2012), el ingreso marginal de nuevas inversiones en una empresa se incrementa si el valor de este ratio es superior a uno, por ende, la inversión realizada hace que esta aumente de valor.

Muchos autores han utilizado la Q de Tobin como dependiente en el cálculo de los modelos en la teoría de la cobertura (Allayannis y Ofek, 2001; Chen y King, 2014; Chen y Shao, 2010; Daske, Hail, Leuz y Verdi, 2008; Lang et al., 1993; Nelson et al., 2005). Estos relacionan el valor que toma la $\mathrm{Q}$ de Tobin frente al uso de derivados financieros por medio de modelos de regresión o por el análisis de series de tiempo y datos de panel. Carter, Rogers y Simkins (2006) observan,

\footnotetext{
${ }^{3}$ Este indicador debe su nombre a James Tobin y William Brainard quienes la introdujeron por primera vez en 1968 en su trabajo titulado«Pitfalls in financial model building» publicado en la revista American Economic Review.
} 
Tabla 1

Descripción de las variables independientes

\begin{tabular}{lll}
\hline Nombre (Nemotécnico) Descripción $\quad$ Unidad de medida
\end{tabular}

Tamaño de la empresa (Size)

Apalancamiento total (Apalanca_Total)

Apalancamiento de largo plazo (Apalanca_LP) Crecimiento de la inversión vía activos fijos (Crecim_Inver_Ac_Neto)

Crecimiento de la inversión vía intangibles (Crecim_Inver_Intang)

Capitalización bursátil (Cap_Bur)

Nivel de exposición (Nivel_Expo)

Diversificación geográfica (Diversifica_Geografica)
Por lo general las empresas grandes son más propensas al uso de derivados que las pequeñas; aun así, existe una evidencia ambigua en la influencia que tiene el tamaño sobre la Q, ya que una empresa con altas expectativas de crecimiento puede presentar una mayor Q que las que tienen alta de madurez

Mide la relación entre los pasivos totales y los activos totales. Literatura previa indica que la relación del apalancamiento puede tener impacto tanto positivo como negativo Mide la relación entre la deuda de largo plazo y los activos totales

Mide la disponibilidad de recursos que las ventas totales asignan al crecimiento de la empresa vía activos fijos, lo que se asocia con oportunidades de crecimiento a futuro y que contribuyen con un mayor valor de la empresa

Mide la disponibilidad de recursos que las ventas totales asignan al crecimiento de la empresa vía activos intangibles, lo que se asocia con actividades de inversión en investigación y desarrollo, y a su vez, con oportunidades de crecimiento a futuro y que contribuyen con un mayor valor de la empresa

Mide el valor de mercado del patrimonio a través del precio de las acciones en circulación a la fecha de corte de cada período

Si una empresa aumenta su participación en mercados extranjeros, aumenta la probabilidad de crecimiento, pero a su vez, estas actividades de comercio internacional van acompañadas de operaciones en moneda extranjera que exponen la estabilidad financiera de las empresas a riesgos del mercado, lo que genera necesidades de coberturas. Por lo tanto, se espera que aquellas empresas que están expuestas a riesgo de tipo de cambio, y usan los derivados con fines de cobertura, aumenten su valor de mercado

Variable dummy que identifica si la empresa está expuesta a riesgos de tipo de cambio
Logaritmo natural de los activos totales

Relación entre Pasivo Total y Activo Total

Relación entre Pasivo no corriente y Activo Total

Relación entre Activo Fijo Neto y las Ventas Totales

Relación entre Activos Intangibles y Ventas Totales

N. ${ }^{\circ}$ acciones en circulación por el precio de cierre

Participación de las exportaciones respecto a las ventas totales de la empresa

Variable dummy que toma el valor de 1 (uno) si la empresa realiza ventas en el extranjero y cero (0) en caso contrario 
Tabla 1 (continuación)

\begin{tabular}{|c|c|c|}
\hline Nombre (Nemotécnico) & Descripción & Unidad de medida \\
\hline $\begin{array}{l}\text { Uso de derivados } \\
\text { (Uso_Derivados) }\end{array}$ & $\begin{array}{l}\text { Se espera que las empresas que realizan } \\
\text { operaciones con derivados como una } \\
\text { estrategia de cobertura, generen mayor valor } \\
\text { a través de la mitigación de los riesgos } \\
\text { asociados a los procesos financieros y } \\
\text { operativos y la disminución en la volatilidad } \\
\text { de los resultados financieros }\end{array}$ & $\begin{array}{l}\text { Variable dummy que toma el valor de } \\
1 \text { (uno) si la empresa utiliza } \\
\text { derivados y cero }(0) \text { en caso contrario }\end{array}$ \\
\hline $\begin{array}{l}\text { Uso de dividendos } \\
\text { (Pago_Dividendos) }\end{array}$ & $\begin{array}{l}\text { El uso de dividendos puede generar efectos } \\
\text { contrarios al aumento del valor de la } \\
\text { empresa; lo anterior debido a que se deja de } \\
\text { reinvertir en proyectos o inversiones futuras } \\
\text { que contribuyen con el crecimiento } \\
\text { corporativo }\end{array}$ & $\begin{array}{l}\text { Variable dummy que toma el valor de } \\
\text { uno (1) si la empresa paga dividendos } \\
\text { y cero (0) en caso contrario }\end{array}$ \\
\hline $\begin{array}{l}\text { Rentabilidad neta } \\
\text { (Rentab_Neta) }\end{array}$ & $\begin{array}{l}\text { Las empresas más rentables tendrán un valor } \\
\text { de mercado más alto al tener flujos de caja } \\
\text { futuros más sostenibles que aquellas } \\
\text { compañías con rentabilidades bajas. La } \\
\text { cobertura que realiza una empresa puede } \\
\text { mitigar el riesgo de altas pérdidas que se ven } \\
\text { reflejadas en la rentabilidad }\end{array}$ & $\begin{array}{l}\text { Relación entre la Utilidad Neta y los } \\
\text { Activos Totales }\end{array}$ \\
\hline $\begin{array}{l}\text { Retorno sobre capital } \\
\text { invertido (ROIC) }\end{array}$ & $\begin{array}{l}\text { Mide el retorno de los activos sobre la } \\
\text { inversión neta. Se espera que, en las } \\
\text { empresas que usan derivados, esta variable } \\
\text { sea mayor que en las empresas que no se } \\
\text { cubren; además, se espera que la volatilidad } \\
\text { disminuya, return on investment capital }\end{array}$ & $\begin{array}{l}\text { Relación entre la utilidad operacional } \\
\text { y los activos netos PPE }\end{array}$ \\
\hline $\begin{array}{l}\text { EBITDA y margen EBITDA } \\
\text { (EBITDA y Mg_EBIDTA) }\end{array}$ & $\begin{array}{l}\text { Mide la capacidad que tiene la empresa de } \\
\text { generar beneficios brutos de explotación o } \\
\text { utilidad operacional }\end{array}$ & $\begin{array}{l}\text { Utilidad operacional más } \\
\text { depreciaciones } \\
\text { Relación entre utilidad operacional } \\
\text { más depreciaciones con respecto a } \\
\text { los ingresos operacionales }\end{array}$ \\
\hline
\end{tabular}

Fuente: elaboración propia.

a partir de la Q de Tobin, la relación del valor de la empresa con la cobertura de combustible en la industria aérea estadounidense entre 1994 y 2000, utilizando mínimos cuadrados generalizados. Por su parte, Montoro en Fierros Villanueva (2012) explica la inversión agregada de las empresas peruanas entre 1999 y 2009, estimando la Q de Tobin con diversas metodologías. En tanto, GómezGonzález, León Rincón y Leiton Rodríguez (2012) evalúan el efecto de la gestión del riesgo y las coberturas sobre el valor de la firma (Q-Tobin) en 81 empresas de Colombia, encontrando que a mayor cobertura, mayor crecimiento del valor de la empresa.

La presente investigación se basa en una metodología simplificada que se referencia en Allayannis y Weston (2001), quienes trabajan en línea con diversos autores como Lewellen y Badrinath (1997), y Ahmed et al. (2014), los cuales se basan, entre otros, en Chung y Pruitt (1994) para considerar la Q de Tobin como la relación entre el valor de mercado de la empresa con respecto a su valor en libros al cierre de año fiscal:

$$
\mathrm{Q}=\frac{\mathrm{VM}(\mathrm{AC})+\mathrm{VL}(\mathrm{PT})}{\mathrm{VL}(\mathrm{TA})}
$$


Donde,

VM $(\mathrm{AC})=$ Capitalización bursátil o valor de mercado del patrimonio.

VL $(T A)=$ Valor en libros del total de activos de la empresa.

VL (PT): Valor en libros del pasivo total.

Con base en lo anterior, se plantean las siguientes hipótesis:

$\mathbf{H}_{1}$ : El uso de los derivados financieros incrementa el valor de las empresas con respecto a aquellas que no los usan.

$\mathbf{H}_{2}$ : Empresas con mayor tamaño, apalancamiento, crecimiento de la inversión, nivel de exposición y rentabilidad se ven compensadas en su valor de mercado por el uso de los derivados.

$\mathbf{H}_{3}$ : Empresas con diversificación geográfica que usan derivados financieros como cobertura incrementan el valor con respecto a aquellas que no.

La tabla 2, panel A, presenta los descriptivos estadísticos generales de las variables principales para toda la muestra completa. Los resultados muestran que el promedio de los activos totales (ingresos operacionales) es de \$3 808578 (\$1 386 729) millones $\mathrm{COP}^{4}$; al igual que en Allayannis et al. (2001), aproximadamente el $51 \%$ de las observaciones de la muestra exportan y participan de operaciones de venta y comercio hacia el extranjero y constituyen un $12 \%$ de los ingresos operacionales que quedan expuestos a riesgos. Y a diferencia de los anteriores, quienes obtienen un $37 \%$, los resultados de este estudio evidencian que el $51 \%$ usa algún instrumento derivado como cobertura. Con respecto a la Q de Tobin, el resultado arrojó un promedio del 1.16, cercano al 1.18 encontrado por Allayannis et al. (2001) y una mediana de 0.86, mientras que en Allayannis et al. (2001) se encontró una mediana de 0.95. El margen EBITDA promedio de la muestra total es del $32 \%$, la rentabilidad neta promedio del $4 \%$ y se pagaron dividendos en un $72 \%$ de la muestra. El apalancamiento promedio total y no corriente del 30 y $11.3 \%$, aproximadamente. El promedio del crecimiento de los activos netos en propiedad, planta y equipo es de 1.89 pesos por cada peso realizado en ventas totales; y el $18.6 \%$ de la muestra representa crecimiento vía intangibles.

El panel B de la tabla 2 refleja resultados promedios con incrementos claros en las variables principales, evidenciando que son empresas más grandes, con mayores ingresos operacionales, de mayor capitalización bursátil y levemente con mayor apalancamiento que el total de la muestra, tanto en pasivo total como de largo plazo, con mayor EBITDA, aunque no mayor margen EBITDA, con una rentabilidad neta y un crecimiento vía intangibles de aproximadamente 2 puntos porcentuales por encima del promedio de la muestra total. Las demás variables disminuyen comparativamente, aunque no lo hacen en grandes proporciones. Como era de esperarse, el porcentaje de empresas que hace coberturas con derivados aumenta aproximadamente en un $24 \%$, y el nivel de exposición al riesgo también se incrementa, casi duplicándose al $24 \%$.

El panel C de la tabla 2 identifica las empresas que no realizan ventas en el extranjero. Claramente este grupo muestra características generales de menor tamaño en la mayoría de sus variables principales; y es de resaltar la baja participación en operaciones de cobertura con derivados, ubicándose 25 puntos porcentuales por debajo de la media total. Sin embargo, refleja mejores indicadores promedio de desempeño financiero como ROIC, margen EBITDA y RSAN, mas no en rentabilidad neta. Finalmente, este grupo muestra que en un $82 \%$ aproximadamente se realiza el pago de dividendos.

Cabe desatacar que no se evidencia una diferencia amplia en el comportamiento promedio de la Q de Tobin entre los tres paneles (A, B y C), conservando su valor promedio muy cercano entre

\footnotetext{
${ }^{4}$ A una tasa de cambio de \$2900 equivalen a USD \$1 313 millones (USD \$478) en Colombia; en Allayannis et al. (2001) el promedio de la muestra es de USD \$7 701 (\$6592) respectivamente.
} 
Tabla 2

Estadísticos descriptivos generales de la muestra

\begin{tabular}{|c|c|c|c|c|c|c|c|c|c|}
\hline & & & & Panel A & : full & & & & \\
\hline Varia & bles & & No. & Mean & & d. Dev. & Min. & & Max. \\
\hline Ac_T & otal & & 975 & 3808578 & & 800000 & 1225 & & 121000000 \\
\hline IngO & & & 975 & 1386729 & & 192551 & 95.18 & & 69100000 \\
\hline Venta & s_Extranjero & & 975 & 205648 & & 43975 & 0 & & 19100000 \\
\hline CapB & & & 975 & 5062570 & & 800000 & 0 & & 224000000 \\
\hline Size & & & 975 & 1314457 & & 6867 & 9414 & 056 & 1860786 \\
\hline Creci & m_Inv_Ac_N & eto & 975 & 1895101 & & 675408 & 0.002 & & 8178028 \\
\hline Q_To & & & 975 & 1160501 & & 3276 & 0.198 & & 4754907 \\
\hline Nivel & Expo & & 975 & 0.1210 & & 2293 & 0 & & 1 \\
\hline Uso_l & Derivados & & 975 & 0.5128 & & 5000 & 0.000 & & 1 \\
\hline Apal & anca_Total & & 975 & 0.2924 & & 2060 & 0.002 & & 1067062 \\
\hline Apal & anca_LP & & 975 & 0.1127 & & 1215 & -0.04 & & 0.7554 \\
\hline Creci & m_Inv_Intan & & 975 & 0.1860 & & 4411 & -0.00 & & 3776087 \\
\hline Cont & & & & & & & & & \\
\hline Rent: & b_Neta & 975 & & 0.0396 & & 184 & -0.534 & & 1641481 \\
\hline Pago & Dividendo & 975 & & 0.7220 & & 482 & 0 & & 1 \\
\hline Diver & sificación & 975 & & 0.5087 & & 001 & 0 & & 1 \\
\hline ROIC & & 975 & & 2415289 & & 33968 & -6977 & 517 & 3938046 \\
\hline EBIT & DA & 975 & & 389253 & & 54554 & -2424 & & 32600000 \\
\hline Mg_F & BITDA & 975 & & 0.3205 & & 471 & -6197 & 799 & 7412946 \\
\hline RSA & & 975 & & 1569938 & & 4579 & -4535 & 386 & 255973 \\
\hline & Pan & el B: Ventas_ & Extranjero $>0$ & & & Pane & C: Ventas_H & xtranjero $=0$ & \\
\hline No. & Mean & Std. Dev. & Min. & Max. & No. & Mean & Std. Dev. & Min. & Max. \\
\hline 496 & 5990173 & 18800000 & 62804 & 121000000 & 479 & 1549557 & 3357173 & 12259 & 17100000 \\
\hline 496 & 2322898 & 8920977 & 13896 & 69100000 & 479 & 417335 & 1276116 & 95.18 & 10100000 \\
\hline 496 & 404247.9 & 1579434 & 9205915 & 19100000 & 479 & 0 & 0 & 0 & 0 \\
\hline 496 & 8581988 & 34200000 & 0 & 224000000 & 479 & 1418245 & 3504364 & 4538349 & 15900000 \\
\hline 496 & 1394698 & 1482492 & 1104779 & 1860786 & 479 & 1231369 & 2064316 & 9414056 & 1665676 \\
\hline 496 & 0.6902 & 0.5412 & 0.0190 & 3239275 & 479 & 314267 & 7890761 & 0.0022 & 8178028 \\
\hline 496 & 1162013 & 1003024 & 0.1988 & 4754907 & 479 & 1158936 & 0.5950 & 0.3063 & 3591698 \\
\hline 496 & 0.2379 & 0.2749 & 0.0000 & 1 & 479 & 0 & 0 & 0 & 0 \\
\hline & 0.7560 & 0.4298 & 0 & 1 & 479 & 0.2609 & 0.4396 & 0 & 1 \\
\hline 496 & 0.3524 & 0.1555 & 0.0480 & 1067062 & 479 & 0.2301 & 0.2319 & 0.0028 & 0.9958 \\
\hline 496 & 0.1410 & 0.1141 & 0.0000 & 0.5164 & 479 & 0.0834 & 0.1221 & -0.0480 & 0.7554 \\
\hline 496 & 0.2160 & 0.4035 & 0.0000 & 3283868 & 479 & 0.1549 & 0.4754 & -0.0097 & 3776087 \\
\hline 496 & 0.0571 & 0.1588 & -0.5343 & 1641481 & 479 & 0.0215 & 0.0427 & -0.3603 & 0.3241 \\
\hline 496 & 0.6229 & 0.4851 & 0 & 1 & 479 & 0.8246 & 0.3806 & 0 & 1 \\
\hline 496 & 1 & 0 & 1 & 1 & 479 & 0 & 0 & 0 & 0 \\
\hline 496 & 0.6073 & 2603703 & -1573209 & 358808 & 479 & 4287425 & 2374665 & -6977517 & 3938046 \\
\hline 496 & 717419.1 & 3125333 & -242407 & 32600000 & 479 & 49441.26 & 122012 & -53172 & 963543 \\
\hline 496 & 0.2995 & 0.4783 & -0.4070 & 3318233 & 479 & 0.3423 & 0.7845 & -6197799 & 7412946 \\
\hline 496 & 0.3947 & 1692407 & -1022586 & 2332252 & 479 & 2786827 & 1543533 & -4535386 & 255973 \\
\hline
\end{tabular}

Fuente: elaboración propia. 
sí (1.1605, 1.1620 y 1.1589, respectivamente) aunque inferior en empresas con ventas netamente locales. Se construyó una Q de Tobin alternativa que incluye el valor de los inventarios declarados en el Balance General, con lo cual se obtuvieron resultados promedios superiores: 10.21 para todo el panel, 1.23 para empresas con ventas en el extranjero y 1.18 para aquellas en las que sus ventas son solo locales; lo que reafirma el concepto de que las empresas que realizan ventas internacionales son empresas más grandes que las que no lo hacen, el cual se ve apoyado por los resultados de las variables tamaño de activos y EBITDA. Los resultados de la media de la Q del panel completo son superiores a la mediana de la Q (0.86), indicando que hay sesgo en la distribución de la Q de Tobin ${ }^{5}$; por lo tanto, se suaviza mediante un proceso de normalización (Q_Tobinw: winsorizada). Adicionalmente, debido a que la muestra está compuesta por empresas de diferentes sectores, se extraen $\mathrm{Q}$ de Tobin promedios sectorizadas que permitan identificar el comportamiento de esta en cada uno de ellos; claramente el sector industrial, que representa el $51 \%$ de la muestra con 500 observaciones de 975 , tiene el valor más bajo (Q_Tobin 0.88). Finalmente, como era de esperarse, el porcentaje de empresas que usan derivados que cuentan con exposición por ventas extranjeras es más alto que aquellas que no usan derivados $(27.7 \%$ contra un $11.4 \%$, ver anexo 1 y anexo 2 para más detalles).

\section{Modelos de datos de panel}

\section{Modelo de regresión de coeficientes constantes}

En este modelo se agrupan todas las observaciones y se analizan de forma consolidada; supone que los coeficientes son iguales para todos los sujetos (empresas analizadas), lo que le da un tratamiento de igualdad a la muestra; así, las variables explicativas no son estocásticas y son estrictamente exógenas, es decir, no depende de los valores actuales, pasados y futuros del termino de error (Gujarati y Porter, 2009). Esta regresión se representa por la siguiente ecuación:

$$
\mathrm{Y}_{i t}=\beta_{1}+\beta_{2} X_{2 i t}+\beta_{3} X_{3 i t}+\beta_{4} X_{4 i t}+\beta_{5} X_{5 i t}+\beta_{6} X_{6 i t}+\beta_{7} X_{7 i t}+\beta_{8} X_{8 i t}+u_{i t}
$$

Donde,

$Y_{i t}=$ Valor de la i-ésima empresa en el t-ésimo momento, logaritmo natural de la Q de Tobin.

$X 2_{i t}=$ Tamaño de la i-ésima empresa en el t-ésimo momento.

$X 3_{i t}=$ Apalancamiento de la i-ésima empresa en el t-ésimo momento.

$X 4_{i t}=$ Crecimiento de la inversión de la i-ésima empresa en el t-ésimo momento.

$X 5_{i t}=$ Diversificación geográfica de la i-ésima empresa en el t-ésimo momento.

$X 6_{i t}=$ Rentabilidad de la i-ésima empresa en el t-ésimo momento.

$X 7_{i t}=$ Uso de derivados por parte de la i-ésima empresa en el t-ésimo momento.

$X 8_{i t}=$ Pago de dividendos de la i-ésima empresa en el t-ésimo momento.

\section{Modelo de efectos fijos}

Bajo este modelo se toma en cuenta la heterogeneidad de las unidades de corte transversal, lo cual asigna a cada una de ellas su propio intercepto, identificando las diferentes características de cada empresa. Las empresas del sector real están sujetas a aspectos propios como la administración, la plataforma estratégica (misión, visión, filosofía, valores y principios, entre otros), la estrategia financiera y los mercados donde tiene presencia. Para Gujarati y Porter (2009), el término efectos fijos se refleja en la invariabilidad que tiene cada intercepto en el tiempo, a pesar de que cada

\footnotetext{
${ }^{5}$ Estos resultados son consistentes con los de Allayannis et al. (2001).
} 
uno de estos se discrimina para cada unidad de corte y transversal. En este caso, la ecuación del modelo de regresión presenta la misma estructura de la fórmula (2) con betas determinadas por:

$$
\beta_{1 i}=\alpha_{1}+\alpha_{2} D_{2 i}+\alpha_{3} D_{3 i}+\ldots+\alpha_{39} D_{39 i}+\alpha_{40} D_{40 i}
$$

Donde,

cada una de las $D_{j i}$ con $j=2, . ., 40$ es una variable dummy para 39 de las 40 empresas, pues la empresa restante estará representada en el intercepto.

\section{Estimador de efectos fijos dentro del grupo}

El objetivo de este modelo es eliminar la heterogeneidad entre las variables (en este caso entre las empresas escogidas como muestra), esto a partir de la diferenciación de las observaciones a través de las medias muestrales; de esta forma, a cada valor individual se le resta su respectiva media muestral dando como resultado valores corregidos por la media.

La ecuación es la siguiente:

$$
y_{i t}=\beta_{2} x 2_{i t}+\beta_{3} x 3_{i t}+\beta_{4} x 4_{i t}+\beta_{5} x 5_{i t}+\beta_{6} x 6_{i t}+\beta_{7} x 7_{i t}+\beta_{8} x 8_{i t}+\varepsilon_{i t}
$$

Donde cada $x k_{i t}$ con $k=2,3, \ldots, 8$ representa la k-ésima variable en diferencias respecto a su media. Lo propio ocurre con la variable dependiente. Algo importante a resaltar en esta última expresión es que el coeficiente del intercepto se elimina, aumentando con ello los grados de libertas de los estadísticos de bondad de ajuste.

\section{Resultados}

Se ajustaron los modelos de regresión en panel de datos mediante varias especificaciones, efectos fijos, efectos aleatorios y efectos aleatorios mediante el estimador de máxima verosimilitud. Al respecto, entre estos dos últimos no se encontró evidencia estadística que indicara que los coeficientes son diferentes, por lo que se eligió el modelo de efectos aleatorios que por defecto el software ajusta ${ }^{6}$.

Las variables fueron sometidas a un proceso de winsorization al $1 \%$ para evitar errores de medida ante la presencia de valores atípicos ${ }^{7}$.

Se estimaron tres tipos de modelos: el primero para el total de empresas, el segundo para las empresas del sector industrial y el tercero para las empresas de los demás sectores (agricultura, comercial, construcción y servicios). Se procedió de esta manera debido a que el sector industrial representa el $51.28 \%$ de la muestra, por lo que para garantizar comparación entre los resultados y evitar sesgos en los mismos, se decidió agregar los demás sectores diferentes al industrial, y ajustar dos modelos sectoriales, uno para el sector industrial y otro para el resto de sectores.

Los resultados de la estimación de los modelos de efectos aleatorios y efectos fijos ${ }^{8}$, para toda la muestra de empresas, se muestran en la tabla 3.

De acuerdo a esta, para la especificación del modelo establecido, las variables que tienen alguna incidencia significativa en el valor de la empresa medida por la Q de Tobin son: el tamaño, el apalancamiento total y de largo plazo, el capital bursátil, el uso de derivados (solo en el modelo

\footnotetext{
${ }^{6}$ El ejercicio de estimación se llevó a cabo mediante la ayuda del software STATA13 MP.

7 Para diferenciar a las variables originales de las winzorizadas se le incluye al final del nombre de cada variable«_W»

${ }^{8}$ Con todas las empresas (panel A), empresas del sector industrial (panel B) y empresas del sector agrícola, construcción y servicios (panel C).
} 
Tabla 3

Modelo con todas las empresas, empresas del sector industrial y empresas del sector agrícola, construcción y servicios

\begin{tabular}{|c|c|c|c|c|c|c|}
\hline \multirow[t]{2}{*}{ Variables } & \multicolumn{2}{|c|}{$\begin{array}{l}\text { Panel A: Todas las } \\
\text { empresas }\end{array}$} & \multicolumn{2}{|c|}{$\begin{array}{l}\text { Panel B: Empresas del } \\
\text { sector industrial }\end{array}$} & \multicolumn{2}{|c|}{$\begin{array}{l}\text { Panel C: Empresas del } \\
\text { sector agrìcola, } \\
\text { construccíón y } \\
\text { servicios }\end{array}$} \\
\hline & $\begin{array}{l}\text { Efectos } \\
\text { aleatorios }\end{array}$ & Efectos fijos & $\begin{array}{l}\text { Efectos } \\
\text { aleatorios }\end{array}$ & Efectos fijos & $\begin{array}{l}\text { Efectos } \\
\text { aleatorios }\end{array}$ & $\begin{array}{l}\text { Efectos } \\
\text { fijos }\end{array}$ \\
\hline \multirow[t]{2}{*}{$\overline{\text { Size_w }}$} & $-0.456^{* * *}$ & $-0.5495^{* * *}$ & $-0.2034^{* * *}$ & $-0.7195^{* * *}$ & $-0.5535^{* * *}$ & $-0.5711^{* * *}$ \\
\hline & -0.036 & -0.0386 & -0.0455 & -0.0775 & -0.0341 & -0.0345 \\
\hline \multirow[t]{2}{*}{ Apalanca_Total_w } & $1.222^{* * *}$ & $1.2135^{* * *}$ & $1.2582^{* * *}$ & $1.3827^{* * *}$ & $1.4627^{* * * *}$ & $1.4582^{* * *}$ \\
\hline & -0.1363 & -0.1362 & -0.2099 & -0.2056 & -0.155 & -0.1563 \\
\hline \multirow[t]{2}{*}{ Apalanca_LP_w } & $-0.4954^{* * *}$ & $-0.5068^{* * *}$ & -0.3723 & -0.2963 & $-0.5797^{* * *}$ & $-0.5859^{* * *}$ \\
\hline & -0.1741 & -0.1719 & -0.2850 & -0.2677 & -0.1808 & -0.1803 \\
\hline \multirow[t]{2}{*}{ Crecim_Inver_Ac_Neto_w } & 0.0006 & 0.0008 & 0.0158 & -0.0061 & 0.0017 & 0.0018 \\
\hline & -0.0037 & -0.0036 & -0.0324 & -0.0303 & -0.0026 & -0.002 \\
\hline \multirow[t]{2}{*}{ Crecim_Inver_Intang_w } & 0.0107 & 0.0342 & $-0.1979^{* *}$ & $-0.1605^{*}$ & $0.0561^{* *}$ & $0.0615^{* *}$ \\
\hline & -0.0333 & -0.0330 & -0.0957 & -0.0894 & -0.0291 & -0.0290 \\
\hline \multirow[t]{2}{*}{ CapBur_w } & $1.26 \mathrm{E}-08^{* * *}$ & $1.22 \mathrm{E}-08^{* * *}$ & $1.25 \mathrm{E}-08$ & $0.000^{* * *}$ & $0.0000^{* * * *}$ & $0.0000^{* * * *}$ \\
\hline & $-1.62 \mathrm{E}-09$ & $-1.65 \mathrm{E}-09$ & $-1.73 \mathrm{E}-09$ & 0 & -8.95 & 0 \\
\hline \multirow[t]{2}{*}{ Nivel_Expo_w } & -0.1696 & -0.2438 & 0.0808 & -0.4298 & -0.5870 & -0.6152 \\
\hline & -0.2302 & -0.2414 & -0.2220 & -0.3086 & -0.3857 & -0.3844 \\
\hline \multirow[t]{2}{*}{ Diversifica_Geografica_w } & 0.0970 & 0.0605 & 0.0880 & 0.0675 & $1.4013^{* *}$ & (omitido) \\
\hline & -0.1085 & -0.1129 & -0.1231 & -0.1335 & -0.5967 & - \\
\hline \multirow[t]{2}{*}{ Uso_Derivados_w } & $0.6369788^{* *}$ & (omitido) & 0.1770 & (omitido) & 0.8458 & (omitido) \\
\hline & -0.2695 & - & -0.1925 & - & -0.5968 & - \\
\hline \multirow[t]{2}{*}{ Pago_Dividendos_w } & -0.0146 & -0.0248 & 0.1108 & 0.0452 & -0.0472 & -0.0483 \\
\hline & -0.0412 & -0.0408 & -0.0705 & -0.0688 & -0.0401 & -0.0399 \\
\hline \multirow[t]{2}{*}{ Rentab_Neta_w } & $0.4650^{* * *}$ & $0.4276^{* * *}$ & $0.4769^{* *}$ & 0.2751 & $1.6054^{* * *}$ & $1.5954^{* * *}$ \\
\hline & -0.1622 & -0.1599 & -0.2081 & -0.1974 & -0.3947 & -0.3928 \\
\hline \multirow[t]{2}{*}{ ROIC_w } & 0.0012 & 0.0008 & $0.0036^{*}$ & 0.0007 & $-0.0834^{* * *}$ & $-0.0838^{* * *}$ \\
\hline & -0.0017 & -0.0016 & -0.0021 & -0.0020 & -0.0135 & -0.0134 \\
\hline \multirow[t]{2}{*}{ Mg_EBITDA_w } & $0.0606^{*}$ & 0.0484 & 0.0962 & 0.0130 & $0.0568^{*}$ & $0.0565^{*}$ \\
\hline & -0.0319 & -0.0316 & -0.0622 & -0.0600 & -0.0299 & -0.0298 \\
\hline \multirow[t]{2}{*}{ _cons } & $6.3961^{* * *}$ & $8.0008^{* * *}$ & $2.9285^{* * *}$ & $10.5011^{* * *}$ & $7.2878^{* * *}$ & $7.97856^{* * *}$ \\
\hline & -0.4652 & -0.5152 & -0.6499 & -1.1174 & -0.4705 & -0.4165 \\
\hline
\end{tabular}

Fuente: elaboración propia.

de efectos aleatorios), la rentabilidad neta y el margen EBITDA (solo en el modelo de efectos aleatorios) $)^{9}$. Los resultados permiten confirmar la primera hipótesis, en tanto que, si una empresa utiliza derivados, incrementa su valor en un $6 \%$ (modelo de efectos aleatorios) y un $8 \%$ (modelo de efectos constantes) con respecto de aquellas que no. Se evidencia además que variables adicionales como el apalancamiento total, la capitalización bursátil, la rentabilidad neta y el margen EBITDA tienen un efecto positivo en la creación de valor, mientras que el tamaño y el apalancamiento de largo plazo tienen un efecto negativo sobre el valor de la empresa. Lo anterior brinda evidencia a favor de algunos aspectos de la hipótesis 2 . Con respecto al tamaño, se confirman los resultados

\footnotetext{
${ }^{9}$ Es importante notar que los resultados de los estimadores no son muy diferentes tanto en magnitud como en el signo de los coeficientes en ambos modelos (efectos aleatorios y efectos fijos), no obstante, el modelo de efectos aleatorios logra capturar otros impactos como los relacionados con el uso de derivados y el margen EBITDA, por lo que se centrará el análisis en sus resultados.
} 
obtenidos con Allayannis et al. (2001) y otros como Lang et al. (1993), cuyo signo también es negativo incluso solo para el grupo de empresas con ventas en el extranjero. Esto es de esperarse debido a que el crecimiento de la empresa vía activos no garantiza que aumente el valor de mercado de la misma. Como se mencionó, los resultados previos con respecto al apalancamiento son variados; en nuestro caso se confirma a Magner Pulgar y Lavín Salazar (2012) y a Bessembinder (1991), en cuanto a que las empresas que usan derivados tienen un mayor apalancamiento frente a las que no (32\% frente a un 26\%); no se analizó si el apalancamiento era en moneda extranjera o local, pero se confirma a Azofra-Palenzuela y Díez Esteban (2001), quienes plantean que la implementación de estrategias de cobertura de riesgos disminuye la probabilidad de enfrentar problemas financieros relacionados con el pago de intereses y de deudas, pues el efecto negativo en el apalancamiento de largo plazo indica que por cada 0.49 pesos que esta disminuye, la empresa gana 1 peso en su valor de mercado.

En el panel B de la tabla 3 se muestran los resultados para la submuestra de empresas del sector industrial. En esta ocasión, las variables que tienen un impacto estadísticamente significativo en el valor de la empresa son: tamaño, apalancamiento total, crecimiento de la inversión vía intangibles, capitalización bursátil (solo en el modelo de efectos fijos) y la rentabilidad neta y el retorno sobre el capital invertido (ambos solo en el modelo de efectos aleatorios). Respecto a las hipótesis 1 y 3 , no es posible inferir nada debido a que las variables uso de derivados y diversificación geográfica no resultan ser estadísticamente significativas en ninguno de los modelos.

Finalmente, en el panel C de la tabla 3, se estiman los modelos de efectos aleatorios y fijos para la submuestra de empresas de los sectores agrícola, comercial, construcción y servicios. En este modelo, las variables estadísticamente significativas son: tamaño, apalancamiento total, apalancamiento largo plazo, crecimiento inversión intangibles, capitalización bursátil, diversificación geográfica (solo en el modelo de efectos aleatorios), rentabilidad neta, retorno sobre el capital invertido y el margen EBITDA. En términos generales, los resultados del panel C son similares a los descritos en los dos modelos anteriores; las principales diferencias son que el crecimiento de la inversión en intangibles es significativo, pero con el signo contrario al hallado en el modelo de las empresas del sector industrial, indicando con ello que, en estas empresas, una buena medida para incrementar su valor es apostarle a la inversión en intangibles cuando se hace cobertura con derivados. Otra diferencia es que, en este modelo, la diversificación geográfica sí tiene efectos significativos sobre el valor de la empresa (o al menos en el estimador de efectos aleatorios, pues en el de efectos fijos el modelo lo omite). Se evidencia que, si una empresa pertenece a este grupo de sectores, y realiza ventas en el extranjero y realiza operaciones con derivados financieros, tendrá un mayor valor de la Q de Tobin, verificando con ello la hipótesis 3 planteada anteriormente. Finalmente, el coeficiente de la variable de retorno sobre el capital invertido presenta un signo diferente al encontrado en el modelo de las empresas del sector industrial. En este caso, el coeficiente es negativo, siendo contrario a lo que se espera, aunque su significancia es del 10\%, lo que podría indicar que, para una muestra más grande de datos, este resultado puede ser diferente.

\section{Discusión y conclusiones}

Los modelos econométricos son una herramienta útil para contrastar hipótesis que la teoría económica, y específicamente la financiera, postulan sobre las variables del entorno macroeconómico y de la empresa. Los modelos de panel de datos permiten hacer una evaluación en dos dimensiones de los fenómenos económicos, ya que cuentan con información de corte transversal y de series de tiempo. En este trabajo se hace uso de este tipo de modelos dado que se cuenta con 
una base de datos conformada por 39 empresas, con información de variables medidas desde el cuarto trimestre del 2008 hasta el cuarto trimestre del 2014.

Se realizó un ejercicio de estimación para verificar si el uso de derivados financieros y otras variables como el tamaño, apalancamiento, crecimiento de la inversión, nivel de exposición a los mercados internacionales, rentabilidad (neta, sobre el capital invertido o sobre activos netos) y la diversificación geográfica logran tener algún efecto sobre la generación de valor de las empresas, medida a través de la Q de Tobin. Para este ejercicio, se dividió el análisis en tres aspectos; el primero involucra al total de empresas de la muestra, el segundo solo a las que pertenecen al sector industrial, y el tercero a las que están en los sectores agrícola, comercial, construcción y servicios. Se encontró evidencia significativa de que el uso de los derivados se asocia positivamente con el valor de mercado de las empresas y genera un valor aproximado del 6.4\% (en la muestra total). De igual modo, se pudo establecer que el apalancamiento total, la capitalización bursátil y la rentabilidad neta tienen un efecto positivo en la generación de valor de las empresas, sin importar a qué sector pertenezcan; adicionalmente se evidenció que, del total de empresas que realizan ventas en el extranjero y usan derivados, el $75 \%$ (375 de 496 empresas) tienen un apalancamiento por debajo de la media del total (un 57\% aproximadamente); en otro sentido, las empresas que no usan derivados pero que realizan ventas en el extranjero se apalancan en un 68\% (11 puntos porcentuales por encima de las primeras; ver anexo 3).

Se encontró que, para las empresas de los sectores agrícola, comercial, construcción y servicios, una buena estrategia para aumentar el valor de la empresa es invertir en activos intangibles; mientras que, para las empresas del sector industrial, una buena medida es procurar aumentar el retorno sobre el capital invertido, pues se evidencia una relación positiva entre este y el valor de la empresa. En cuanto a la diversificación geográfica, se observa un resultado esperado al relacionarse positivamente con el valor de mercado de las compañías analizadas; esta es una ventaja competitiva en términos de conocimiento, oportunidad y diversificación de objetivos, pero incrementa la exposición al riesgo, que deberá ser mitigada con estrategias de coberturas a través del mercado de derivados. Por último, el pago de dividendos muestra una relación positiva con el valor de mercado en todos los modelos, resultado que no se esperaba debido a que, a mayor pago de dividendos se ven limitadas futuras inversiones en la empresa. Se evidencian dos situaciones generales: la primera, que las empresas grandes, y que además exportan, participan en mayor medida del mercado de derivados que las pequeñas, bien sea por su tamaño, su experiencia o madurez, o porque pueden acceder más fácil a los mercados internacionales, a diferencia de las pequeñas; la segunda es que la participación en el mercado de derivados en Colombia aún no es muy alta; esto puede atribuirse al desconocimiento de los derivados y las estrategias de cobertura a utilizar, a decisiones de la compañía por optar por cobertura natural como estrategia, porque los costos de transacción son altos o porque solo es de interés de algunos sectores. Futuros trabajos podrían procurar aumentar el tamaño de la muestra de los sectores agrícola, comercial, construcción y servicios con el fin de hacer una estimación puntual sobre los posibles efectos que las variables consideradas en este estudio pueden tener en el valor de las empresas de tales sectores. Se recomienda utilizar otras medidas de tamaño, o ajustar relaciones no lineales para esta variable y así establecer si el signo negativo se debe a solo un valor promedio de esta, o si efectivamente si las empresas son micro, pequeñas, medianas o grandes empresas tienen efectos diferenciados en el valor de la empresa. 


\section{Anexo 1. Estadístico de la $Q$ de Tobin por sectores}

\begin{tabular}{llllll}
\hline Sectores & Obs. & Mean & Std.Dev. & Min. & Max. \\
\hline Agropecuario & 275 & 1.4767 & 0.8889 & 0.3651 & 4.3216 \\
Comercial & 25 & 1.4815 & 0.2466 & 0.9137 & 1.9381 \\
Construcción & 75 & 1.3710 & 0.3529 & 0.4889 & 2.0761 \\
Industrial & 500 & 0.8885 & 0.6512 & 0.1988 & 4.7549 \\
Servicios & 100 & 1.4131 & 1.2378 & 0.3138 & 3.7694 \\
Total - promedio & 875 & 1.3261 & 0.6755 & 0.4561 & 3.3720 \\
\hline
\end{tabular}

Anexo 2. Porcentaje de empresas que usan (no usan) derivados y tienen algún nivel de exposición

\begin{tabular}{llllll}
\hline Usan derivados & Obs. & Mean & Std.Dev. & Min. & Max. \\
\hline No & 121 & 0.1140 & 0.0822 & 0.000518 & 0.6 \\
Sí & 375 & 0.2779 & 0.3022 & 0.0018945 & 1 \\
\hline
\end{tabular}

Anexo 3. Porcentaje de empresas que usan (no usan) derivados vs. apalancamiento total

\begin{tabular}{llllll}
\hline Usan derivados & Obs. & Mean & Std.Dev. & Min. & Max. \\
\hline No & 121 & 0.6859 & 0.4660 & 0 & 1 \\
Sí & 375 & 0.5733 & 0.4952 & 0 & 1 \\
\hline
\end{tabular}

\section{Referencias}

Ahmed, H., Azevedo, A. y Guney, Y. (2014). European Financial Management Association 2104 (pp. 1-5), 44, Disponible en: http:/www.efmaefm.org/OEFMAMEETINGS/EFMA ANNUAL MEETINGS/2014-Rome/papers/EFMA2014_ 0342_fullpaper.pdf

Allayannis, G. y Ofek, E. (1998). Exchange rate exposure hedging and the use of foreign currency derivatives (June 1997). Disponible en: http://papers.ssrn.com/abstract=1296392

Allayannis, G. y Ofek, E. (2001). Exchange rate exposure, hedging, and the use of foreign currency derivatives. Journal of International Money and Finance, 20(2), 273-296. http://dx.doi.org/10.1016/S0261-5606(00)00050-4

Allayannis, G., Lel, U. y Miller, D. P. (2012). The use of foreign currency derivatives, corporate governance, and firm value around the world. Journal of International Economics, 87(1), 65-79. http://dx.doi.org/10.1016/j.jinteco.2011.12.003

Allayannis, G. y Weston, J. P. (2001). The use of foreign currency derivatives and firm market value. Review of Financial Studies, 14(1), 243-276. http://dx.doi.org/10.1093/rfs/14.1.243

Alvarado Cardozo, N., Rassa Robayo, J. S. y Rojas Moreno, J. S. (2014). Caracterización del Mercado de Derivados Cambiarios en Colombia. Borradores de Economia. Banco de La República, 860, 45. Disponible en: http://www.banrep.gov.co/sites/default/files/publicaciones/archivos/be_860.pdf

Azofra-Palenzuela, V. y Díez Esteban, J. M. (2001). La cobertura corporativa del riesgo de cambio en las empresas no financieras españolas. Nuevas Tendencias en Dirección de Empresas, 6, 1-21. Disponible en: https://www.researchgate.net/profile/Valentin_Azofra/publication/5200902_La_cobertura_corporativa_del_riesgo_de _cambio_en_las_empresas_no_financieras_espanolas/links/00b49522fb74cded73000000/La-cobertura-corporativadel-riesgo-de-cambio-en-las-empresas-no

Bartram, S. M., Brown, G. W. y Conrad, J. (2011). The effects of derivatives on firm risk and value source. The Journal of Financial and Quantitative Analysis, 46(4), 967-999. http://dx.doi.org/10.1017/S0022109011000275 
Bartual Sanfeliu, C. y Moya Clemente, I. (2008). Relación entre la creación o no de valor y la información económico-financiera de las empresas. pp. 83. En J. J. Pindado García y G. Payne (Eds.), Estableciendo puentes en una economía global Escuela Superior de Gestión Comercial y Marketing, ESIC. Disponible en: https://dialnet.unirioja.es/servlet/articulo?codigo=2724555\&info=resumen\&idioma=S

Bessembinder, H. (1991). Forward contracts and firm value: Investment incentive and contracting effects incentive and contracting effects source. The Journal of Financial and Quantitative Analysis, 26(4), 519-532. http://dx.doi.org/10.2307/2331409

Caballero, R. J. (2003). The future of the IMF and the world bank. The American Economic Review, 93(2), 31-38. Disponible en: http://www.jstor.org/stable/3132196

Carter, D. A., Rogers, D. A. y Simkins, B. J. (2006). Does hedging affect firm value? Evidence from the US airline industry. Financial Management, 35(1), 53-86. Disponible en: http://www.jstor.org/stable/30134004.

Chen, J. y King, T. H. D. (2014). Corporate hedging and the cost of debt. Journal of Corporate Finance, 29, 221-245. http://dx.doi.org/10.1016/j.jcorpfin.2014.09.006

Chen, Y. y Shao, Q. Q. (2010). 2010 International Conference on Management Science and Engineering, ICMSE 2010. Melbourne, Australia: IEEE. http://dx.doi.org/10.1109/ICMSE.2010.5719968

Chung, K. y Pruitt, S. (1994). A simple approximation of Tobin's Q. Financial Management, 23(3), 70-74. http://www.jstor.org/stable/3665623

Daske, H., Hail, L., Leuz, C. y Verdi, R. (2008). Mandatory IFRS reporting around the world: Early evidence on the economic consequences. Journal of Accounting Research, 46(5), 1085-1142. http://dx.doi.org/10.1111/j.1475-679X.2008.00306.x

DeMarzo, P. y Duffie, D. (1995). Corporate incentives for hedging and hedge accounting. Review of Financial Studies, 8(3), 743-771. http://www.jstor.org/stable/2962238?origin=JSTOR-pdf

Dufey, G. y Srinivasulu, S. L. (1983). The case for corporate management of foreign exchange risk. Financial Management, 12(4), 54-62. http://www.jstor.org/stable/3665269

Fierros Villanueva, P.A. (2012). El mercado de derivados financieros y su impacto en el valor de las empresas en México [tesis de maestría]. Tijuana, México: El Colegio de la Frontera Norte.

Froot, K. A., Scharfstein, D. S. y Stein, J. C. (1993). Risk management: Coordinating corporate investment and financing policies. The Journal of Finance, 48(5), 1629-1658. http://dx.doi.org/10.1111/j.1540-6261.1993.tb05123.x

Géczy, C., Minton, B. y Schrand, C. (1997). Why firms use currency derivatives. The Journal of Finance, 52(4), $1323-1354$. http://dx.doi.org/10.1111/j.1540-6261.1997.tb01112.x

Gómez-González, J. E., León Rincón, C. E. y Leiton Rodríguez, K. J. (2012). Does the use of foreign currency derivatives affect firms' market value? Evidence from Colombia. Emerging Markets Finance and Trade, 48(4), 50-66. http://dx.doi.org/10.2753/REE1540-496X480403

Gómez González, J.E., León, C. y Leiton Rodríguez, K.J. (2009, May). Does the use of foreign currency derivatives affect Colombian firm's market value? Borradores de Economía (562), 26. Disponible en: http://www.banrep.gov.co/es/contenidos/publicacion/does-use-foreign-currency-derivatives-affect-colombian-firmsmarket-value

Gujarati, D. y Porter, D. (2009). Econometría. México: Mc Graw Hill.

Hammoudeh, S. y McAleer, M. (2013). Risk management and financial derivatives: An overview. The North American Journal of Economics and Finance, 25, 109-115. http://dx.doi.org/10.1016/j.najef.2012.06.014

Jin, Y. y Jorion, P. (2006). Oil and gas producers. The Journal of Finance, 61(2), 893-919. Disponible en: http://www.jstor.org/stable/3699361

Kapitsinas, S. K. (2008). The Impact of derivatives usage on firm value: Evidence from Greece, 10947. Disponible en: http://mpra.ub.uni-muenchen.de/id/eprint/.

Lang, L.H. P., Stulz, R.M., Fama, G., Grinblatt, M., Hirshleifer, D., Hubbard, G., .. Scheinkman, J. (1993). Tobin's Q corporative diversification and firm performance.

Lewellen, W. G. y Badrinath, S. G. (1997). On the measurement of Tobin's q. Journal of Financial Economics, 44(1), 77-122. http://dx.doi.org/10.1016/S0304-405X(96)00013-X

Magner Pulgar, N. y Lavín Salazar, J. (2012). ¿Son los derivados financieros una herramienta de gestión de riesgo de uso frecuente en la industria de agronegocios? Revista Latinoamericana de Administración, 51, 65-78. Disponible en: http://revistaacademia.uniandes.edu.co/index.php/Cladea/article/view/583/629

Modigliani, F. y Miller, M. (1958). The cost of capital, corporation finance and the theory of investment. The American Economic Review, 48(3), 261-297. Disponible en: http://www.jstor.org/stable/1809766

Montoro, C. y Navarro, A. (2010). Estimación de la Q de Tobin para la economía peruana. Revista Estudios Económicos, $19,33-45$. 
Munoz Martinez, H. (2016). Hedging neoliberalism: Derivatives as state policy in Mexico. New Political Economy, 21(3), 291-304. http://dx.doi.org/10.1080/13563467.2016.1113947

Nelson, J. M., Moffitt, J. S. y Affleck-Graves, J. (2005). The impact of hedging on the market value of equity. Journal of Corporate Finance, 11(5), 851-881. http://dx.doi.org/10.1016/j.jcorpfin.2005.02.003

Sierra González, J. H. y Londoño Bedoya, D. A. (2010). Cobertura con derivados en empresas manufactureras colombianas: análisis previo a la apertura del mercado de derivados en la bolsa de valores de Colombia. Cuadernos de Administración, 23(41), 237-260. Disponible en: http://search.ebscohost.com/login.aspx?direct=true\&db=fua\&AN=59510022\&lang=es\&site=ehost-live

Smith, C. y Stulz, R. (1985). The determinants of firm's hedging policies. Journal of Financial and Quantitive Analysis, 20(4), 391-406. http://www.jstor.org/stable/2330757

Vivel Búa, M., Otero González, L., Fernández López, S. y Durán Santomil, P. (2015). Is value creation consistent with currency hedging? The European Journal of Finance, 21(10-11), 912-945. http://dx.doi.org/10.1080/1351847X.2013.773262 